\title{
Erratum to: Diet and exercise in uterine cancer survivors (DEUS pilot) - piloting a healthy eating and physical activity program: study protocol for a randomized controlled trial
}

Dimitrios A. Koutoukidis' ${ }^{1}$ Rebecca J. Beeken², Ranjit Manchanda ${ }^{1,3,4}$, Matthew Burnell', M. Tish Knobf ${ }^{1,5}$ and Anne Lanceley ${ }^{1 *}$

\section{Erratum}

Upon publication of the article [1], it was noticed that Table 1 was missing some times in the 4th column of 'Session 2'. This information has now been included in this erratum.

\section{Author details \\ 'Department of Women's Cancer, EGA Institute for Women's Health, University College London, London, UK. ${ }^{2}$ Health Behaviour Research Centre, Department of Epidemiology \& Public Health, University College London, London, UK. ${ }^{3}$ Department of Gynaecological Oncology, Barts Health NHS Trust, Royal London Hospital, London, UK. ${ }^{4}$ Barts Cancer Institute, Queen Mary University of London, London, UK. ${ }^{5}$ Acute Care/Health Systems Division, Yale University School of Nursing, New Haven, CT, USA.}

Received: 4 January 2017 Accepted: 5 January 2017

Published online: 18 January 2017

\section{Reference}

1. Koutoukidis DA, Beeken RJ, Manchanda R, Burnell M, Knobf MT, Lanceley A.

Diet and exercise in uterine cancer survivors (DEUS pilot) - piloting a healthy

eating and physical activity program: study protocol for a randomized

controlled trial. Trials. 2016;17:130. doi:10.1186/s13063-016-1260-1.

\footnotetext{
*Correspondence: a.lanceley@ucl.ac.uk

'Department of Women's Cancer, EGA Institute for Women's Health,

University College London, London, UK

Full list of author information is available at the end of the article
} 
Table 1 Structure and content of the Shape-up following cancer treatment sessions

\begin{tabular}{ll}
\hline Session 2 Keeping to a regular eating pattern & Review: Discussion about self-monitoring and food diaries, and goal progress \\
& Volunteer-led discussion: Keeping to a regular eating pattern \\
& Key learning points: The importance of keeping to a regular eating pattern, \\
& the definition of a regular eating pattern, the importance of breakfast, \\
& suggestions for goals, disadvantages of eating regularly. \\
& Break \\
& New topic: Goals and rewards \\
& Discussion about the principles of goal-setting, group exercise about setting \\
& SMART goals, exercise about goal planning, discussion about rewards, \\
& and group exercise about non-food rewards \\
& Round-up, and preparation for next session \\
& Take home message \\
&
\end{tabular}

